\title{
Corrigendum: Efficient genome engineering by targeted homologous recombination in mouse embryos using transcription activator-like effector nucleases
}

Daniel Sommer, Annika E. Peters, Tristan Wirtz, Maren Mai, Justus Ackermann, Yasser Thabet, Jürgen Schmidt, Heike Weighardt, F. Thomas Wunderlich, Joachim Degen, Joachim L. Schultze \& Marc Beyer

Nature Communications 5:3045 doi: 10.1038/ncomms4045 (2014); Published 13 Jan 2014; Updated 20 Feb 2015

In the original version of this Article, the middle initial of the author Annika E. Peters was omitted from the author information. This has now been corrected in both the PDF and HTML versions of the Article. 